\section{Synergistic Effect of Radiofrequency Electromagnetic Fields of Dental Light Cure Devices and Mobile Phones Accelerates the Microleakage of Amalgam Restorations: An in vitro Study}

\author{
Mortazavi S. M. J.,2, Dehghani Nazhvani A.3,4, Paknahad \\ M. $5,6 *$
}

\begin{abstract}
Background: Previous studies have shown that exposure to electromagnetic fields produced by magnetic resonance imaging or mobile phones can lead to increased microleakage of dental amalgam.
\end{abstract}

Objective: The aim of the present study was to investigate the effect of electromagnetic field of a commercial dental light cure device and a common GSM mobile phone on microleakage of amalgam restorations.

Materials and Methods: Identical class $\mathrm{V}$ cavities were prepared on the buccal surfaces of 60 non-carious extracted human teeth. The samples were randomly divided into 4 groups of 20 samples each. The samples in the first group were not exposed to electromagnetic fields, while the second and the third groups were exposed to electromagnetic fields produced by a commercial light cure device, or mobile phone radiation ( $60 \mathrm{~min}$ ), respectively. The fourth group was exposed to electromagnetic radiations emitted by both mobile phone for $60 \mathrm{~min}$ and light cure device. Then, teeth samples were scored for microleakage according to a standard dye penetration protocol by examination under a stereomicroscope.

Results: The mean score of microleakage in the fourth group (light cure + mobile phone) was significantly higher than that of the control group $(\mathrm{P}=0.030)$. Moreover, the scores of microleakage in this group were significantly higher than that of the second group (light cure only) $(\mathrm{P}=0.043)$.

Conclusion: Exposure of amalgam restorations to electromagnetic fields produced by both light cure devices and mobile phones can synergistically increase the microleakage of amalgam restorations.

\section{Keywords}

Light Cure Device, Amalgam, Microleakage, Electromagnetic Radiation, Mobile Phones

\section{Introduction}

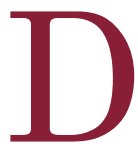
ental amalgam has widely been used for more than 150 years for restoring posterior teeth because of many advantages such as easy manipulation, low technique sensitivity, highly wearing resistance, low cost, durability and insolubility in oral fluids [1-4]. However, one of the most important drawbacks of amalgam restorations is lack of chemical adhesion to the tooth structure which can result
${ }^{1}$ Ionizing and Non-ionizing Radiation Protection

Research Center, Shiraz

University of Medical Sci-

ences, Shiraz, Iran

${ }^{2}$ Medical Physics and

Medical Engineering

Department, School of

Medicine, Shiraz Univer-

sity of Medical Sciences

Shiraz, Iran

${ }^{3}$ Biomaterial Research

Center, School of Den-

tistry, Shiraz University

of Medical Sciences,

Shiraz, IR Iran

${ }^{4}$ Oral and Maxillofacial

Pathology Department,

School of Dentistry, Shi-

raz University of Medical

Sciences, Shiraz, IR Iran

${ }^{5}$ Oral and Dental Dis-

ease Research Center,

School of Dentistry, Shi-

raz University of Medical

Sciences, Shiraz, IR Iran

${ }^{6}$ Department of Oral and Maxillofacial Radiology, School of Dentistry, Shiraz University of Medical Sciences, Shiraz, IR Iran

*Corresponding author: M. paknahad,

D.Ds. Oral and Maxillofacial Radiology Department, Shiraz Dental

School, Ghasrodasht

Street, Shiraz, Iran

E-mail: paknahadmaryam@yahoo.com

Received: 25 June 2016 Accepted: 12 July 2016 
in amalgam microleakage. Microleakage is defined as the penetration of microorganisms and toxins between the restoration and the walls of cavity preparation. Microleakage can cause tooth discoloration around the margins of restorations, secondary caries, restoration failure, postoperative sensitivity, pulpal pathology and partial or total loss of restoration $[1,5,6]$.

Many home electrical appliances and dental devices generate low frequency magnetic fields. These electromagnetic fields especially in electric appliances used near the head such as electric shavers, hairdryers, electric toothbrushes and light curing devices can induce electric current in dental metallic appliances and may cause problems. Over the past several years, our laboratories at the Ionizing and Non-ionizing Radiation Protection Research Center (INIRPRC) have expanded their focus on studying the health effects of exposure to some common and/or occupational sources of electromagnetic fields (EMFs) such as cellular phones [7-15], mobile base stations [16], mobile phone jammers [17-19], laptop computers [20], radars [8], dentistry cavitrons [21], MRI $[22,23]$, Wi-Fi routers $[24,25]$ and different coils [26, 27]. In dental clinics, light-cured resins polymerized by curing light sources, have been reported to produce low-frequency electromagnetic fields, are also widely used for both convenience and speed [11]. Furthermore, the worldwide increase in mobile phone use has caused great concerns about the possible detrimental effects of microwave radiations emitted by these communication devices. Therefore, in this study, we first aimed to investigate the effects of exposure to lowfrequency magnetic fields generated by light cure units and mobile phones on amalgam microleakage.

\section{Material and Methods}

Teeth Samples

Eighty non-carious extracted premolar and molar teeth were selected. The fractured or defected teeth were excluded. After cleaning and surface debridement, the teeth were stored in saline solution for up to 3 months. Identical class $\mathrm{V}$ cavities ( $3 \mathrm{~mm}$ length, $5 \mathrm{~mm}$ wide, $2 \mathrm{~mm}$ deep) were prepared on the buccal surfaces at the cement-enamel junction using carbide burs (SS White Burs, Lakewood, NJ) and a high speed turbine with air-water spray using a template. To ensure cutting efficiency, a separate bur was used after every 6 cavity preparations. The cavities were restored with Cinalux (non-gama-2, spherical amalgam, Faghihi Dental, Tehran, Iran) amalgam. After triturating according to manufacturers' directions, the amalgams were condensed incrementally towards the cavity walls using small condensers. All the procedures for restoration of cavities including cavity preparation, burnishing and polishing were performed by the same clinician. The restored teeth were placed in saline solution at $37^{\circ} \mathrm{C}$ for seven days.

\section{Exposure of Samples}

The samples were randomly divided into 4 groups of 20 samples each. The samples in the first group were not exposed to electromagnetic fields (control group), while the second and the third groups were exposed to electromagnetic fields produced by a commercial light cure device (Blue Light LA500, APOSA Enterprise Co., Taiwan), and mobile phone radiation (60 $\mathrm{min}$ ), respectively. In this study, a GSM $900 \mathrm{MHz}$ mobile phone simulator was used. This simulator was developed by the Department of Medical Physics and Biomedical Engineering, School of Medicine, Shiraz University of Medical Sciences, Shiraz, Iran with the cooperation of private sector. During exposure, the temperature of samples was monitored. Samples were placed in the far field distance from GSM antenna to obtain uniform irradiation. The average $S A R=\sigma|E|^{2} / \rho$ for the samples was calculated. The fourth group was exposed to electromagnetic radiations emitted by both mobile phone (60 min) and light cure 
device.

\section{Post-Exposure Treatment}

After exposure, all specimens were placed in $2 \%$ basic fuchsin solution. Then, teeth samples were sectioned and scored for microleakage according to a standard dye penetration protocol by examination under a stereomicroscope.

\section{Microleakage Evaluation}

Two layers of nail varnish were applied to the entire teeth surfaces except for the restorations and $1 \mathrm{~mm}$ around them. The specimens were immersed in $2 \%$ basic fuchsin dye solution (merck, Germany) at the room temperature for 24 hours and then they were rinsed in tap water and dried. A slow speed water cooled saw was used to section each tooth buccolingually. The section corresponding to the central portion of the tooth restoration was examined at the gingival, axial and occlusal margins under a stereomicroscope (Olympus. Tokyo, Japan) at $80 \times$ magnification by the examiner who was blinded to the groups. The degree of microleakage was evaluated according to a standard ranking in which [6] $0=$ No dye penetration; $1=$ Dye penetration along the enamel; $2=$ Dye penetration along the dentine-enamel junction

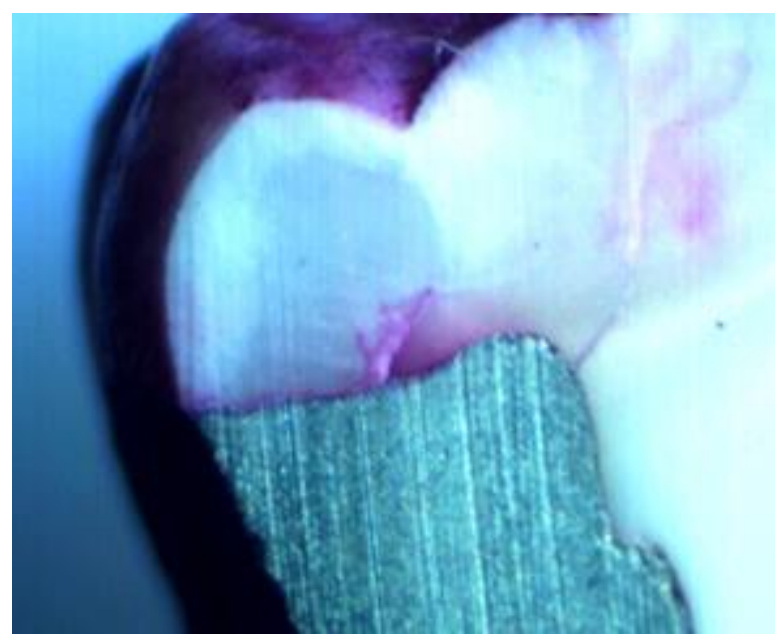

Figure 1: Dye penetration through enamel, passing DEJ to dentin (score 3 ) in a sample tooth
(DEJ) but not including the axial wall and $3=$ Dye penetration along the axial wall. (Figures 1,2)

The data were analyzed using Mann-Whitney U-test to compare microleakage in the case and control groups to identify any statistically significant differences at the significance level of 0.95 ( $p$ value $<0.05$ ).

\section{Results}

The findings of this study are summarized in Table 1. As it is indicated in this table, the relative frequency of teeth samples with no dye penetration (Score 0) was 75\% for non-exposed control samples, while the frequencies for those samples exposed only to light cure or mobile phone were $60 \%$ and $50 \%$, respectively. This frequency for the teeth samples exposed to both of these radiations was as low as $30 \%$. The mean score of microleakage in the fourth group (light cure + mobile phone) was significantly higher than that of the control group $(\mathrm{P}=0.030)$. Moreover, the scores of microleakage in this group was significantly higher than that of the second group (light cure only) ( $\mathrm{P}=0.043)$. However, these scores were not significantly different in the third (mobile phone only) and fourth (light cure + mobile phone) groups.

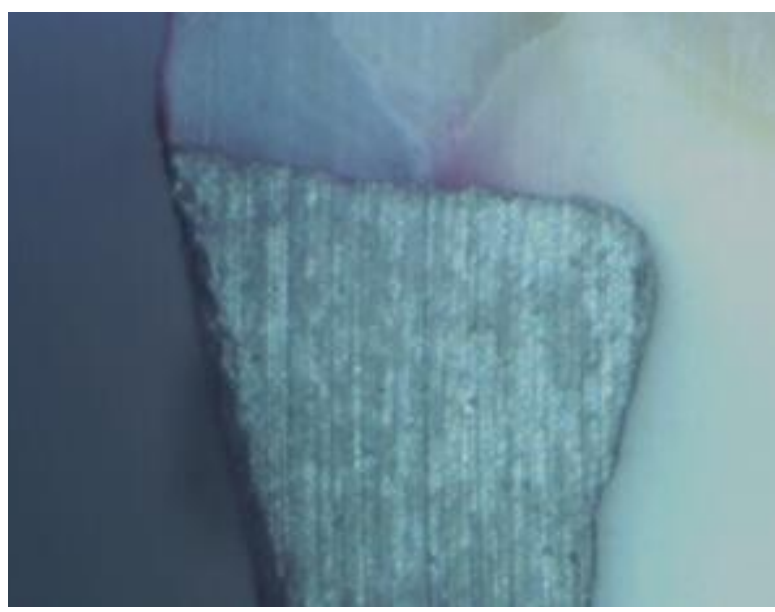

Figure 2: No Dye leakage (score 0) in a control tooth 
Table 1: The distribution of the scores in the control and exposure groups.

\begin{tabular}{lcccc} 
& \multicolumn{5}{c}{ Percent (\%) of the scores } \\
\cline { 2 - 5 } Exposure/ (Group) & $\mathbf{0}$ & $\mathbf{1}$ & $\mathbf{2}$ & $\mathbf{3}$ \\
\hline No Exposure ( Group1) & 75 & 5 & 5 & 15 \\
\hline Only Light Cure (Group 2) & 60 & 20 & 15 & 5 \\
\hline Only Mobile Phone (Group 3) & 50 & 25 & 15 & 10 \\
\hline Light Cure + Mobile Phone (Group 4) & 30 & 20 & 30 & 20
\end{tabular}

\section{Discussion}

Altogether, the findings of this study showed that exposure of the teeth samples with amalgam restorations to either electromagnetic fields produced by light cure devices or those generated by mobile phones can lead to increased microleakage of amalgam restorations. Interestingly, it was also shown that exposure of the amalgam restorations to both of these radiations (light cure + mobile phone) induces a synergistic response which further increases the microleakage of amalgam restorations. In this study, we did not assess the release of mercury but it can be hypothesized that this kind of synergism may be observed for the release of mercury from dental amalgam fillings after exposure to electromagnetic radiation. In this light, further experiments are needed to clarify the health effects of this phenomenon.

Nowadays, many electrical dental appliances including personal devices such as electric toothbrushes and professional dental devices such as curing lights for light-cured dental resins are widely used because of their convenience. The electric circuit powers generate low-frequency electromagnetic waves that can induce electric currents in metallic objects in the oral cavity. Thus, the effects of these induced currents on human health have been of concern particularly because of their great proximity to the brain. Moreover, drastically increased number of mobile phone users during recent years has generated great concern too. Mortazavi et al. reported that radiofrequency radiation emitted from mobile phones significantly increased mercury release from amalgam restorations [13]. Increase in the microleakage of dental amalgam restorations following exposure to electromagnetic fields of MRI has been reported in previous studies [28, 29]. Shahidi et al. have suggested that the increase in microleakage following MRI might be attributed to the thermoelectromagnetic convection induced by exposure to EMFs which was supposed to be responsible for the enhancement of diffusion process, grain boundary migration and vacancy formation resulting in microleakage. However, Mortazavi and Paknahad believed that the magnitude of temperature increase is not high enough to justify this theory [30]. On the other hand, Akgun OA et al. did not indicate any statistically significant differences in the extent of microleakage in groups with or without MRI exposure [31]. Our team also found that PEMF exposure generated by Helmholtz coil does not have adverse effects on microleakage of amalgam restorations. Moreover, we have recently found that that $\mathrm{X}$-ray exposure increased microleakage of amalgam restorations [32]. In the present study, it was revealed that amalgam microleakage was not significantly different in the light cure exposed group and mobile phone exposed group compared to the control group. However, microleakage was significantly higher in teeth exposed to both radiofrequency radiations emitted from mobile phone and light cure device.

It is worth noting that today mobile phones are much more frequently used for surfing the Internet than calling. In the present study, the 
authors have limited their experiments only to 2 modes (i.e. talk and off modes). Therefore, further investigations on the effect of other remaining modes of "standby" and "Talk+WiFi" (talking using a mobile phone that is connected to Wi-Fi network) on microleakage of amalgam restoration are necessary.

\section{Conclusion}

This was the first study which assessed the effect of exposure to radiofrequency of light cure devices and mobile phones on the microleakage of amalgam restorations. We theorized that exposure to radiofrequency heating and electromagnetic field of light cure device do not increase the microleakage of amalgam restorations. Therefore, regarding the significance of this challenging issue, further in vitro and in vivo studies are necessary to confirm this contention.

\section{Acknowledgment}

The authors thank the Vice-chancellery of Shiraz University of Medical Sciences for supporting this research. In addition, the authors thank Dr. Vosoughi from the Dental Research Development Center for the statistical analysis. This study was supported by the Ionizing and Non-ionizing Radiation Protection Research Center (INIRPRC), Shiraz University of Medical Sciences (SUMS), Shiraz, Iran.

\section{Conflict of Interest}

The authors declared no conflict of interest.

\section{References}

1. Bembi S, Bembi NN, Sood A, Gambhir A. To Evaluate the Effect of Different Adhesive Materials on the Microleakage of Bonded Amalgam Restorations: An in vitro Study. Int J Clin Pediatr Dent. 2012;5:185-9. doi.org/10.5005/jp-journals-10005-1163. PubMed PMID: 25206165. PubMed PMCID: 4155881.

2. Opdam NJ, Bronkhorst EM, Loomans BA, Huysmans MC. 12-year survival of composite vs. amalgam restorations. J Dent Res. 2010;89:1063-7. doi.org/10.1177/0022034510376071. PubMed PMID: 20660797.

3. Mitchell RJ, Koike M, Okabe T. Posterior amalgam restorations--usage, regulation, and longevity. Dent
Clin North Am. 2007;51:573-89. doi.org/10.1016/j. cden.2007.04.004. PubMed PMID: 17586144.

4. Cenci MS, Piva E, Potrich F, Formolo E, Demarco FF, Powers JM. Microleakage in bonded amalgam restorations using different adhesive materials. Braz Dent J. 2004;15:13-8. doi.org/10.1590/ S0103-64402004000100003. PubMed PMID: 15322639.

5. Morrow LA, Wilson NH, Setcos JC, Watts DC. Microleakage of amalgam cavity treatment systems: an in vitro evaluation. Am J Dent. 2002;15:262-7. PubMed PMID: 12572646.

6. Burgess J0, Walker R, Davidson JM. Posterior resin-based composite: review of the literature. Pediatr Dent. 2002;24:465-79. PubMed PMID: 12412962.

7. Mortazavi SM, Motamedifar M, Namdari G, Taheri M, Mortazavi AR, Shokrpour N. Non-linear adaptive phenomena which decrease the risk of infection after pre-exposure to radiofrequency radiation. Dose Response. 2014;12:233-45. doi.org/10.2203/ dose-response.12-055.Mortazavi. PubMed PMID: 24910582. PubMed PMCID: 4036396.

8. Mortazavi SM, Taeb S, Dehghan N. Alterations of visual reaction time and short term memory in military radar personnel. Iran $J$ Public Health. 2013;42:428-35. PubMed PMID: 23785684. PubMed PMCID: 3684731.

9. Mortazavi SM, Rouintan MS, Taeb S, Dehghan N, Ghaffarpanah AA, Sadeghi Z, et al. Human shortterm exposure to electromagnetic fields emitted by mobile phones decreases computer-assisted visual reaction time. Acta Neurol Belg. 2012;112:171-5. doi.org/10.1007/s13760-012-0044-y. PubMed PMID: 22426673.

10. Mortazavi S, Mosleh-Shirazi M, Tavassoli A, Taheri M, Mehdizadeh A, Namazi S, et al. Increased Radioresistance to Lethal Doses of Gamma Rays in Mice and Rats after Exposure to Microwave Radiation Emitted by a GSM Mobile Phone Simulator. Dose Response. 2013;11:281-92. doi.org/10.2203/ dose-response.12-010.Mortazavi. PubMed PMID: 23930107. PubMed PMCID: 3682203.

11. Mortazavi S, Mosleh-Shirazi M, Tavassoli A, Taheri $\mathrm{M}$, Bagheri Z, Ghalandari R, et al. A comparative study on the increased radioresistance to lethal doses of gamma rays after exposure to microwave radiation and oral intake of flaxseed oil. Iranian Journal of Radiation Research. 2011;9:9-14.

12. Mortavazi S, Habib A, Ganj-Karami A, Samimi-Doost R, Pour-Abedi A, Babaie A. Alterations in TSH and Thyroid Hormones following Mobile Phone Use. Oman Med J. 2009;24:274-8. doi.org/10.5001/ omj.2009.56. PubMed PMID: 22216380. PubMed PMCID: 3243874.

13. Mortazavi SM, Daiee E, Yazdi A, Khiabani K, Kavousi $A$, Vazirinejad $R$, et al. Mercury release from 
dental amalgam restorations after magnetic resonance imaging and following mobile phone use. Pak J Biol Sci. 2008;11:1142-6. doi.org/10.3923/ pjbs.2008.1142.1146. PubMed PMID: 18819554.

14. Mortazavi SM, Ahmadi J, Shariati M. Prevalence of subjective poor health symptoms associated with exposure to electromagnetic fields among university students. Bioelectromagnetics. 2007;28:32630. doi.org/10.1002/bem.20305. PubMed PMID: 17330851.

15. Mortazavi S, Motamedifar M, Namdari G, Taheri M, Mortazavi A. Counterbalancing immunosuppression-induced infections during long-term stay of humans in space. Journal of Medical Hypotheses and Ideas. 2013;7:8-10. doi.org/10.1016/j. jmhi.2012.12.001.

16. Mortazavi S. Safety issue of mobile phone base stations. J Biomed Phys Eng. 2013;3:1-2.

17. Mortazavi S, Parsanezhad M, Kazempour M, Ghahramani $P$, Mortazavi A, Davari M. Male reproductive health under threat: Short term exposure to radiofrequency radiations emitted by common mobile jammers. J Hum Reprod Sci. 2013;6:124-8. doi. org/10.4103/0974-1208.117178. PubMed PMID: 24082653. PubMed PMCID: 3778601.

18. Rafati A, Rahimi S, Talebi A, Soleimani A, Haghani M, Mortazavi SM. Exposure to Radiofrequency Radiation Emitted from Common Mobile Phone Jammers Alters the Pattern of Muscle Contractions: an Animal Model Study. J Biomed Phys Eng. 2015;5:133-42. PubMed PMID: 26396969. PubMed PMCID: 4576874.

19. Shekoohi Shooli F, Mortazavi SA, Jarideh S, Nematollahii S, Yousefi F, Haghani M, et al. Short-Term Exposure to Electromagnetic Fields Generated by Mobile Phone Jammers Decreases the Fasting Blood Sugar in Adult Male Rats. J Biomed Phys Eng. 2016;6:27-32. PubMed PMID: 27026952. PubMed PMCID: 4795326.

20. Mortazavi SMJ, Tavassoli A, Ranjbari F, Moammaiee P. Effects of laptop computers' electromagnetic field on sperm quality. Journal of Reproduction \& Infertility. 2010;11(4).

21. Mortazavi SM, Vazife-Doost S, Yaghooti M, Mehdizadeh S, Rajaie-Far A. Occupational exposure of dentists to electromagnetic fields produced by magnetostrictive cavitrons alters the serum cortisol level. J Nat Sci Biol Med. 2012;3:60-4. doi. org/10.4103/0976-9668.95958. PubMed PMID: 22690053. PubMed PMCID: 3361780.

22. Mortazavi SM, Neghab M, Anoosheh SM, Bahaeddini N, Mortazavi G, Neghab P, et al. High-field MRI and mercury release from dental amalgam fillings. Int J Occup Environ Med. 2014;5:101-5. PubMed PMID: 24748001.

23. Mortazavi SM, Daiee E, Yazdi A, Khiabani K, Ka- vousi $A$, Vazirinejad $R$, et al. Mercury release from dental amalgam restorations after magnetic resonance imaging and following mobile phone use. Pak J Biol Sci. 2008;11:1142-6. doi.org/10.3923/ pjbs.2008.1142.1146. PubMed PMID: 18819554.

24. Mahmoudi R, Mortazavi S, Safari S, Nikseresht M, Mozdarani $H$, Jafari $M$, et al. Effects of microwave electromagnetic radiations emitted from common Wi-Fi routers on rats' sperm count and motility. Int $J$ Radiat Res. 2015;13:363-8.

25. Paknahad M, Mortazavi SM, Shahidi S, Mortazavi $G$, Haghani M. Effect of radiofrequency radiation from Wi-Fi devices on mercury release from amalgam restorations. J Environ Health Sci Eng. 2016;14:12. doi.org/10.1186/s40201-016-0253-z. PubMed PMID: 27418965. PubMed PMCID: 4944481.

26. Haghnegahdar A, Khosrovpanah $H$, AndishehTadbir A, Mortazavi G, Saeedi Moghadam M, Mortazavi $S$, et al. Design and fabrication of helmholtz coils to study the effects of pulsed electromagnetic fields on the healing process in periodontitis: preliminary animal results. J Biomed Phys Eng. 2014;4:83-90. PubMed PMID: 25505775. PubMed PMCID: 4258865.

27. Paknahad M, Shahidi S, Mortazavi SMJ, Mortazavi G, Moghadam MS, Nazhvani AD. The Effect of Pulsed Electromagnetic Fields on Microleakage of Amalgam Restorations: An in Vitro Study. Shiraz E-Medical Journal. 2016;17(2).

28. Shahidi SH, Bronoosh P, Alavi AA, Zamiri B, Sadeghi AR, Bagheri MH, et al. Effect of magnetic resonance imaging on microleakage of amalgam restorations: an in vitro study. Dentomaxillofac Radiol. 2009;38:470-4. doi.org/10.1259/dmfr/30077669. PubMed PMID: 19767518.

29. Yilmaz S, Misirlioglu M. The effect of $3 \mathrm{~T} \mathrm{MRI}$ on microleakage of amalgam restorations. Dentomaxillofac Radiol. 2013;42:20130072. doi. org/10.1259/dmfr.20130072. PubMed PMID: 23674614. PubMed PMCID: 3756742.

30. Mortazavi SM, Paknahad M. Effect of magnetic resonance imaging on microleakage of amalgam restorations: an in vitro study. Dentomaxillofac Radiol. 2016;45:20150187. doi.org/10.1259/ dmfr.20150187. PubMed PMID: 26224142.

31. Akgun OM, Polat GG, Turan Illca A, Yildirim C, Demir $P$, Basak F. Does magnetic resonance imaging affect the microleakage of amalgam restorations? Iran J Radiol. 2014;11:e15565. PubMed PMID: 25763074. PubMed PMCID: 4341166.

32. Paknahad M, Nazhvani AD, Jarideh S, Mozdarani $H$, Mortazavi G, Haghani M, et al. Increased Mercury release due to exposure to electromagnetic radiation as a limiting factor for using dental amalgam. Int J Radiat Res. 2016;14:355-9. 